\title{
Pain and combat injuries in soldiers returning from Operations Enduring Freedom and Iraqi Freedom: Implications for research and practice
}

\author{
Michael E. Clark, PhD; ${ }^{1-2 *}$ Matthew J. Bair, MD, MS; ${ }^{3}$ Chester C. Buckenmaier III, MD; ${ }^{4}$ Ronald J. Gironda, \\ PhD; ${ }^{1-2}$ Robyn L. Walker, PhD $^{1}$ \\ ${ }^{1}$ James A. Haley Veterans' Hospital, Tampa, FL; ${ }^{2}$ University of South Florida, Tampa, FL; ${ }^{3}$ Center of Excellence on \\ Implementing Evidence-Based Practice, Richard L. Roudebush Department of Veterans Affairs Medical Center, India- \\ napolis, IN; ${ }^{4}$ Department of Anesthesiology, Walter Reed Army Medical Center, Washington, DC
}

\begin{abstract}
Operation Enduring Freedom (OEF) and Operation Iraqi Freedom (OIF) have resulted in a growing number of seriously injured soldiers who are evacuated to the United States for comprehensive medical care. Trauma-related pain is an almost universal problem among these war-injured soldiers, and several military and Department of Veterans Affairs initiatives have been implemented to enhance pain care across the continuum of medical services. This article describes several innovative approaches for improving the pain care provided to OEF and OIF military personnel during acute stabilization, transport, medical-surgical treatment, and rehabilitation and presents summary data characterizing the soldiers, pain management services provided, and associated outcomes. We also identify some of the pain assessment, classification, and treatment challenges emerging from work with this population and provide recommendations for future research and practice priorities.
\end{abstract}

Key words: acute pain, chronic pain, combat injuries, pain assessment, polytrauma, postacute pain, regional anesthesia, rehabilitation, trauma, traumatic brain injury.

\section{INTRODUCTION}

Operation Enduring Freedom (OEF) and Operation Iraqi Freedom (OIF) commenced in October 2001 and May 2003, respectively, as part of the global war on terror. To date, more than 1 million military personnel have been deployed to Afghanistan or Iraq. Approximately
21,000 of those deployed have been wounded in combat, and about 46 percent of these casualties have been medically evacuated (http://www.icasualties.org). Blast-related injuries predominate; wounds from improvised explosive devices (IEDs), land mines, shrapnel, and other blast phenomena account for 65 percent of combat injuries [1]. Approximately 60 percent of these injured soldiers have symptoms of traumatic brain injury (TBI) (http:// www.dvbic.org/). Nevertheless, despite the number of casualties and the wound severity, the U.S. military medical system has been remarkably successful in the management of combat trauma during these conflicts, as

Abbreviations: $\mathrm{CPNB}=$ continuous peripheral nerve block, IED = improvised explosive device, MARAA = Military Advanced Regional Anesthesia and Analgesia, MTF = military treatment facility, OEF = Operation Enduring Freedom, OIF = Operation Iraqi Freedom, PCA = patient-controlled analgesia, PRC = Polytrauma Rehabilitation Center, PTSD $=$ posttraumatic stress disorder, $\mathrm{RA}=$ regional anesthesia, $\mathrm{SD}=$ standard deviation, $\mathrm{TBI}=$ traumatic brain injury, VA $=$ Department of Veterans Affairs, WRAMC $=$ Walter Reed Army Medical Center.

*Address all correspondence to Michael E. Clark, PhD; James A. Haley Veterans' Hospital, Chronic Pain Rehabilitation Program, 2CW, 13000 Bruce B. Downs Blvd, Tampa, FL 33612; 813-972-2000, ext 7484; fax: 813-978-5867.

Email: michael.clark8@va.gov 
evidenced by the $>90$ percent survival rate following injury [2]. This success has been attributed to multiple factors, including improved body armor [3-4], surgical care deployed far forward on the battlefield [5], and rapid evacuation to major hospitals via military aircraft equipped with sophisticated medical equipment [6]. The advantages of these innovations for survival following combat trauma are clear, but this success has created other unforeseen medical challenges. One of the most significant has been the management of pain immediately following combat trauma, during subsequent acute medical treatment, and throughout initial rehabilitation efforts.

In this article, we introduce the subject of traumarelated pain and describe several innovative approaches for improving the pain care provided to OEF and OIF military personnel during acute stabilization, transport, medical-surgical treatment, and rehabilitation. Next, we identify some of the pain assessment, classification, and treatment challenges emerging from work with this population and, where possible, provide associated clinical practice suggestions. Finally, we close with a discussion of related research priorities. Throughout, we should note that empirical data in this area, while provocative, are limited. Therefore, the conclusions and implications we provide should be viewed as tentative and subject to modification as additional data become available.

\section{PAIN AND TRAUMA}

Because trauma patients are often seriously injured and have multiple body regions involved, they commonly experience significant levels of pain [7-8]. Since the initial care of the trauma patient involves stabilization of cardiovascular and respiratory status, employment of life-saving procedures (e.g., chest tube, endotracheal intubation), and assessment of multiple and severe injuries, pain may not be appropriately assessed and thus not adequately treated [9]. Compounding the problem of pain treatment in the trauma setting are provider concerns that opioids may lead to hypotension, respiratory depression, clouding of mental status, and obfuscation of changes in clinical status [7].

When adequate pain control is not achieved, individuals suffer unnecessarily, adding to concerns about their ability to recover and function normally after the trauma. Unfortunately, most trauma-care algorithms, even at major trauma centers, do not include a systematic approach to pain assessment. Furthermore, empirical studies of pain assessment in trauma patients are virtually nonexistent. This lack is unfortunate given that pain may be constantly present for trauma patients from the time of the initial injury through the acute treatment phase and may continue into rehabilitation and the return to the community [10].

Edwards has categorized the continuum of trauma care into three phases: (1) emergency, (2) acute or healing, and (3) rehabilitation [10]. The emergency phase begins at the time of injury and ends after resuscitation and emergency stabilization are complete. This phase is frequently accompanied by intense pain from the initial traumatic insult and nociceptive input from damaged tissue. The acute or healing phase starts after emergency stabilization and ends when all surgical treatment of injuries is complete. The rehabilitation phase follows and continues until function is restored. In this article, we use a modified version of Edwards' tripartite categorization of trauma care to organize our discussion of pain among soldiers with OEF and OIF combat casualties. Our adaptation emphasizes the importance of psychosocial factors, particularly during the rehabilitation phase of treatment.

\section{EMERGENCY PHASE: PAIN CARE FOLLOWING COMBAT TRAUMA AND DURING TRANSPORT}

The severity of injuries suffered by OEF and OIF soldiers is unprecedented. The blasts caused by suicide bombers and IEDs in particular often result in multiple visible (tissue wounds) and hidden (hearing loss, confusion) injuries [11]. Table 1 presents summary surgical data from the U.S. Army Regional Anesthesia and Pain Management Initiative Combat Casualty Database, which was developed as part of a process-improvement program at the Walter Reed Army Medical Center (WRAMC) in Washington, DC, one of the nation's primary military treatment facilities (MTFs). These data pertain to 1,565 field and follow-up surgeries performed on 287 severely injured OEF and OIF soldiers who were medically evacuated to WRAMC. As illustrated in the table, orthopedic and soft tissue injuries predominated, which reflects the high frequency of blast injuries. The number of surgeries conducted (an average of almost 5.5 surgeries per person) highlights the multiplicity and severity of the combat wounds. Not included are additional surgeries performed at later stages of recovery or during rehabilitation. Nor are hidden physical or emotional 
Table 1.

Distribution of 1,565 surgeries on soldiers with combat injuries according to type of procedure and location (field [ $n=931$ ] or Walter Reed Army Medical Center [WRAMC] $[n=634])$.

\begin{tabular}{|c|c|c|}
\hline Procedure & Field & WRAMC \\
\hline Orthopedic & 290 & 265 \\
\hline Amputation & 114 & 40 \\
\hline Amputation Revision & - & 55 \\
\hline External Fixation & 104 & 11 \\
\hline Internal Fixation & 39 & 85 \\
\hline Joint Exploration & 13 & 15 \\
\hline Joint Fixation/Manipulation & - & 8 \\
\hline Joint Reconstruction/Replacement & - & 9 \\
\hline Bone Grafting & 1 & 2 \\
\hline Hardware Removal & - & 11 \\
\hline Ligament Repair & - & 10 \\
\hline Nerve Repair/Neurolysis & 3 & 8 \\
\hline Tendon Repair/Transfer & 6 & 11 \\
\hline Other & 10 & - \\
\hline Soft Tissue & 249 & 330 \\
\hline Exploration/Debridement \pm VAC & 219 & 287 \\
\hline Foreign Body Removal & 14 & 1 \\
\hline Burn Wound Care & 9 & - \\
\hline Skin Grafting or Flap Coverage & 7 & 30 \\
\hline Scar Release & - & 5 \\
\hline Wound Closure & - & 7 \\
\hline Vascular & 82 & 1 \\
\hline Major Repair & 32 & - \\
\hline Fasciotomy & 34 & - \\
\hline Other & 16 & 1 \\
\hline Thoracic & 24 & 1 \\
\hline Tube Thoracostomy & 14 & - \\
\hline Pericardiotomy & 3 & - \\
\hline Other & 7 & 1 \\
\hline Abdominal Exploration & 23 & 0 \\
\hline Solid Organ Injury Repair & 4 & - \\
\hline Hollow Viscera Repair/Diversion & 4 & - \\
\hline Other & 17 & - \\
\hline Head and Neck & 32 & 37 \\
\hline Other & 32 & 37 \\
\hline Unknown & 19 & - \\
\hline
\end{tabular}

injuries, which often become evident during the rehabilitation stage, reflected in these data. Nonetheless, these data unequivocally demonstrate the need for effective paincontrol strategies following initial combat trauma.

Unfortunately, efforts to provide adequate pain control following significant combat injuries are complicated by the vast and complex military medical treatment and evacuation chain. Following severe injuries, soldiers will receive immediate field hospital care so the extent of their injuries can be assessed and the emergency care needed to stabilize their condition can be provided. Subsequently, they are evacuated to MTFs in the United States. During the evacuation, they rapidly travel thousands of miles, come in contact with scores of healthcare providers from different military services, and pass through multiple hospital systems. While this swift initial treatment and evacuation strategy contributes significantly to improved trauma survival rates, it poses multiple challenges for the 
effective control of trauma-related pain. In prior conflicts, wounded soldiers spent many days to weeks recovering from wounds in field hospitals before they were considered sufficiently stable for transport to major hospitals outside of the operational theater. Morphine was an effective pain-control tool in this environment because patients remained confined to a field hospital where their pain could be managed with scheduled doses of morphine and the adverse effects (respiratory depression, nausea, vomiting, etc.) could be closely monitored [12]. In the present conflicts, evacuation of the wounded has been greatly accelerated with wounded soldiers arriving in a major military hospital (i.e., Landstuhl Regional Medical Center, Germany) in some cases $<24$ hours postinjury. Seriously wounded soldiers who likely would not have survived in previous wars are now routinely saved because of rapid air evacuation to the United States via critical-care Aeromedical Transport Teams [6]. In this environment, the goal of field medicine is stabilization of the patient for rapid transport to the next level of care and traditional intravenous morphine pain protocols have limited usefulness. Evacuation flights are often crowded with patients, light conditions are low, vibration and noise are high, monitoring resources are constrained, and healthcare personnel are limited. The need for new pain management technologies and strategies that could effectively treat pain without increasing the burden on healthcare workers was recognized early in the OEF and OIF conflicts.

Fortuitously, military anesthesiologists began working on initiatives to improve battlefield perioperative care well before the onset of hostilities in OEF and OIF. One successful initiative focused on improving regional anesthesia (RA) training in the military and further developing RA for the battlefield. RA techniques employ peripheral nerve stimulation and/or ultrasound to accurately place needles in the proximity of target nerves or a nerve plexus. Local anesthetic is then injected to block the conduction of these nerves, which results in loss of motor, sensory, and autonomic function distal to the block. The surgical block will last for many hours and protect the patient from pain and the surgical stress response since afferent input from the blocked region is attenuated. With slightly larger needles, continuous peripheral nerve block (CPNB) can be established by placing a perineural catheter next to the blocked nerves. Continuous infusions of more dilute local anesthetic then can be infused to maintain analgesia (with return of motor function) to the blocked area for days to weeks.
Early efforts of the Army Regional Anesthesia and Pain Management Initiative focused on the role of RA and CPNB in the anesthetic and analgesic management of patients in austere environments [13]. This experience, as well as the work of early military anesthesiologists who demonstrated the positive aspects of RA in previous conflicts [14], led to the placement of the first CPNB catheter in an American soldier on October 7, 2003, in Balad, Iraq [15]. Many patients' pain has been managed with CPNB following this initial success and, with assistance from the Military Advanced Regional Anesthesia and Analgesia (MARAA) committee established in 2005, the CPNB infusion pump was subsequently approved for in-flight use. Today, CPNB is a viable alternative for the anesthetic and analgesic management of soldiers wounded in combat.

Another pain-management challenge was the lack of patient-controlled analgesia (PCA) pumps for use in combat support hospitals or military evacuation flights. This limitation was particularly disturbing since the advantages of PCA technology are well validated; it has been used safely for decades in the civilian population [16]. With PCA, a small microprocessor-driven pump delivers on-demand doses of opioids to a patient based on limits programmed by a physician. This technology frees the nurse from manual administration of opioids, while empowering the patients with control over their own pain medication delivery. The MARAA committee considered PCA technology particularly promising for morphine delivery during air transport. After a review of available infusion pump technology, the MARAA committee recommended a modified commercial infusion pump as a temporary triservice solution for PCA pending a final pump selection. Today, these devices are being used by wounded soldiers throughout their evacuation. The recent addition of PCA technology to medical evacuation offers a multimodal approach to battlefield pain control that meets or exceeds modern civilian standards [17].

\section{ACUTE PHASE: PAIN MANAGEMENT DURING MILITARY TREATMENT FACILITY CARE}

Following transport to the United States, OEF and OIF evacuees are admitted to MTFs across the country. These individuals often have multiple severe battle wounds and disabling pain and are treated with advanced pain-treatment technologies. Table 2 presents some descriptive information extracted from the combat casualty database on 287 medical 
evacuees treated with RA at WRAMC. As indicated in the table, multiple-limb orthopedic injuries predominated in these wounded soldiers, which correlates with other authors' findings $[4,18]$. The high incidence of polytrauma with multiple orthopedic injuries is also notable. The 287 soldiers with combat casualties underwent 634 operations with advanced RA for anesthesia and perioperative analgesia during their hospitalization at WRAMC. Primary indications for RA were surgery ( $n=$ $580)$, pain control $(n=40)$, and surgery combined with pain control $(n=14)$. Individuals with combat casualties required multiple operations (mean \pm standard deviation [SD] = $2 \pm 1$ surgeries/patient [range 1-9]) during prolonged hospital stays at WRAMC (mean \pm SD $=25 \pm$ 15 days [range 2-148]) [19]. The capability of CPNB to provide long-term analgesia and surgical anesthesia during frequent trips to the operating room is a significant benefit of this particular pain-management technique. In this patient cohort, 361 CPNB catheters were placed and used for a mean \pm SD of $9 \pm 5$ days (range 1-34), which represents more than 1,718 patient-catheter days [19]. Pain visual-analog-scale scores, which were available for a subset of 126 individuals, declined significantly from a pre-CPNB mean of 3.7 to a post-CPNB mean of 2.2 [19]. The average length of time CPNB was used to manage pain in these polytrauma patients, compared with the 1-3 days typical of other medical-surgical cohorts [2021], attests to its efficacy.

This technique also provides effective anesthesia for multiple dressing changes and surgical debridements, along with days to weeks of analgesia. Overall, the data indicate that advanced pain-management technologies are viable alternatives or adjuvants to pharmaceutical methods of pain control during medical-surgical care for OEF and OIEF combat wounded.

Table 2.

Characteristics of patients' combat injuries that were treated with regional anesthetic blocks $(n=287)$. Data presented as frequency, $n(\%)$, unless otherwise noted.

\begin{tabular}{lcc}
\hline \multicolumn{1}{c}{ Characteristic } & $\begin{array}{c}\text { Male } \\
(\mathbf{n = 2 6 9 )}\end{array}$ & $\begin{array}{c}\text { Female } \\
(\boldsymbol{n}=\mathbf{1 8})\end{array}$ \\
\hline $\begin{array}{l}\text { Age (yr) (mean } \pm \text { standard deviation) } \\
\text { Injury Mechanism }\end{array}$ & $28.1 \pm 0.5$ & $27.5 \pm 1.7$ \\
Blast or Fragment & & $6(33.3)$ \\
Bullet & $164(61.0)$ & $1(5.6)$ \\
Motor Vehicle Accident & $38(14.1)$ & $3(16.7)$ \\
Other & $11(4.1)$ & $2(11.1)$ \\
Unknown & $12(4.4)$ & $6(33.3)$ \\
Injury Distribution & $44(16.4)$ & $15(83.3)$ \\
Orthopedic & & $2(11.1)$ \\
Polytrauma & $189(70.3)$ & $1(5.6)$ \\
Other Single Site & $72(26.8)$ & $1(5.6)$ \\
Orthopedic Injuries & $8(2.9)$ & $7(38.9)$ \\
0 & & $4(22.2)$ \\
1 & $7(2.6)$ & $4(22.2)$ \\
2 & $124(46.1)$ & $2(11.1)$ \\
3 & $86(31.9)$ & $7(38.9)$ \\
$>3$ & $33(12.3)$ & $5(27.8)$ \\
Number of Injury Sites & $19(7.1)$ & $3(16.7)$ \\
1 & & $3(16.7)$ \\
2 & $82(30.5)$ & \\
3 & $71(26.4)$ & \\
\hline \hline
\end{tabular}




\section{REHABILITATION PHASE: PAIN AND POLYTRAUMA CARE}

Given the severity and complexity of OEF and OIF combat wounds, the Secretary of the Department of Veterans Affairs (VA) designated four Polytrauma Rehabilitation Centers (PRCs) to serve as the primary VA receiving facilities for veterans and active duty military with polytrauma. Polytrauma was defined as “. . . two or more injuries to physical regions or organ systems, one of which may be life threatening, resulting in physical, cognitive, psychological, or psychosocial impairments and functional disability" [22]. Because the majority of those with polytrauma also exhibit symptoms of TBI, PRCs were located at facilities with established TBI lead centers. The mission of the PRCs is to provide inpatient rehabilitation treatment, ongoing medical-surgical care, and family support to soldiers with polytrauma.

The James A. Haley Veterans' Hospital PRC, Tampa, Florida (Tampa PRC), has been actively treating OIF and OEF combat-related polytraumatic injuries since 2003. The Tampa PRC has an interdisciplinary approach for the comprehensive assessment and delivery of care that targets the entire range of impairments and needs of the combat wounded and their families. The Tampa PRC aims to restore the person to his or her maximal physical, functional, psychological, and vocational status and to facilitate his or her entry into the appropriate social, familial, or treatment setting following rehabilitation. Pain management is recognized as an important component of rehabilitative care, particularly among those with traumatic injuries. Consistent with this perspective, a pain psychologist attends polytrauma multidisciplinary rounds, serves as a full member of the clinical care and administrative teams, provides a full range of treatment services to polytrauma patients, and offers consultative and educational services to staff.

To date, the Tampa PRC has participated in the rehabilitation of 50 OEF or OIF soldiers with polytraumatic combat-related injuries. We reviewed the medical records of these wounded soldiers to obtain information regarding their presenting problems and treatment during their initial inpatient rehabilitation stay at the Tampa PRC. All procedures were approved by the University of South Florida Institutional Review Board and the Tampa VA Research and Development committee. Demographic, medical, pain, and treatment data for these individuals with combat casualties who were experiencing pain were extracted from the record and are presented here.

Demographic information for this group of warinjured is summarized in Table 3. As illustrated in the table, all but one of the persons treated was male. The mean \pm SD age of these soldiers was $28.9 \pm 8.7$ years and the mean \pm SD number of years of education was $12.6 \pm$ 1.1. Most of the soldiers in this sample were deployed to Iraq (98\%) on active duty status, and their mean \pm SD length of deployment was $5.3 \pm 3.2$ months. At the time of discharge from the Tampa PRC, most soldiers returned home but remained on active duty status (85\%) with 30 or more days of convalescence leave. The vast majority of these soldiers will be returning to the Tampa PRC, other VA PRCs, or to other MTFs for further surgeries, interventions, and rehabilitation necessitated by their injuries.

Table 3.

Demographic data for injured soldiers treated at James A. Haley Veterans’ Hospital Polytrauma Rehabilitation Center, Tampa, Florida $(n=50)$.

\begin{tabular}{lc}
\hline \multicolumn{1}{c}{ Characteristic } & $\begin{array}{c}\text { Mean } \pm \text { Standard } \\
\text { Deviation or \% }\end{array}$ \\
\hline Sex & 98 \\
Male & 2 \\
Female & $28.9 \pm 8.7$ \\
Age (yr) & \\
Race/Ethnicity & 82 \\
Caucasian & 8 \\
African American & 8 \\
Hispanic & 2 \\
American Indian & 56 \\
Marital Status & 40 \\
Married & 4 \\
Never Married & $12.6 \pm 1.1$ \\
Divorced & \\
Education (yr) & 40 \\
Military Service Branch & 34 \\
Army & 18 \\
Marines & 8 \\
Navy & \\
National Guard & 78 \\
Duty Status Before Deployment & 22 \\
Active Duty & $5.3 \pm 3.2$ \\
Active Reservist & \\
Deployment Length (mo) & \\
Deployment Theater & \\
Iraq & \\
Afghanistan & \\
Other Territories & \\
\hline \hline
\end{tabular}


Approximately 80 percent of these wounded service members incurred some type of combat-related TBI (penetrating $=58 \%$, closed $=22 \%$ ). All sustained multiple traumas among which orthopedic injuries (50\%) were the most common, followed by soft-tissue damage (48\%), hearing problems (48\%), and eye injuries (44\%). Orthopedic injuries treated at the Tampa PRC often involved fractures or crush injuries, while the most common soft-tissue damage was from multiple location shrapnel wounds. Other, less common, traumatic injuries in this sample included burns (12\%), compartment syndromes resulting in fasciotomies (12\%), limb amputations (12\%), and spinal cord injuries (10\%). Overall, the mean \pm SD number of injury categories (including TBIs) per soldier treated at the Tampa PRC was $3.3 \pm 1.2$ (range 2-6). However, these numbers do not reflect multiple injuries sustained within each injury category (i.e., multiple fractures, numerous soft-tissue injuries, or more than one amputation site). As a result, the actual average number of injuries per soldier was higher. The most common causes of injury in this sample were IEDs (70\%), airborne shrapnel (26\%), mortar rounds (22\%), and gunshot wounds (6\%). Of the 50 soldiers admitted to the Tampa PRC, 15 sustained injuries from more than one source. Cognitive limitations were quite common among the soldiers treated. The mean \pm SD Rancho Los Amigos Scale score at admission was $5.2 \pm 1.9$ and the mean \pm SD Glasgow Coma Scale score was $9.2 \pm 3.9$. Approximately 71 percent of this sample had Rancho scores $<7$ (moderate-to-severe TBI) upon admission and 76 percent had Glasgow scores <13 (moderate-to-severe TBI). The high percentage of patients with scores in the moderateto-severe impairment range suggests that significant cognitive impairment characterized the majority of this sample.

Records revealed that 48 (96\%) of these soldiers experienced at least one pain problem during their inpatient rehabilitation stay. Despite the frequency and severity of the described cognitive impairments, reliable and valid pain-intensity ratings were obtained from 34 (68\%) of the soldiers upon admission. For this sample, the mean \pm SD pain-intensity rating at admission was $5.6 \pm 2.2$ (range 1-10) and the mean \pm SD duration of pain at admission was $83.7 \pm 152.7$ days (range 24-1,054). Approximately 70 percent of soldiers with pain experienced it in more than one site (mean $\pm \mathrm{SD}=2.3 \pm 1.4$ ). The most common primary and secondary pain site was the head (32\% and $22 \%$, respectively). Additional information regarding primary and secondary pain sites is included in Table 4.
Table 4.

Primary and secondary pain locations.

\begin{tabular}{lc}
\hline Location & \% \\
\hline Primary Pain & 32 \\
Head & 23 \\
Leg(s) & 13 \\
Shoulder(s) & 11 \\
Arm(s) & 6 \\
Hand(s) & 4 \\
Neck & \\
Secondary Pain & 22 \\
Head & 16 \\
Low Back & 14 \\
Face & 14 \\
Hand(s) & 12 \\
Leg(s) & 10 \\
Neck & 10 \\
Abdomen &
\end{tabular}

Pain-related problems were common among the wounded, with impairments in physical (42\%) and emotional (34\%) function most prominent. To best manage these complex pain problems during the rehabilitation process, clinicians employed multiple pain-treatment methods. The most common method was medication management (100\%), followed by physical $(40 \%)$ and occupational (38\%) therapies. Table 5 presents detailed information regarding all reported pain-related impairments and documented pain-management interventions used for this sample of polytrauma patients. As noted in the table, a psychologist provided individual cognitivebehavioral therapy for pain management to 13 percent of the total sample of injured soldiers. This relatively low percentage is an artifact of the initial lack of availability of an appropriate provider. Since a pain psychologist has been available, approximately 25 percent of the injured soldiers experiencing pain problems receive individual cognitive-behavioral therapy.

The most frequently prescribed pain medications at admission were opioids (58\%), followed by nonsteroidal anti-inflammatory medications (50\%), and anticonvulsants (20\%). Nonsteroidal and anticonvulsant medication use increased by the time of discharge, while 10 percent of those on opioids at intake no longer required them at discharge. This result is consistent with the efforts of the Tampa PRC medical team to reduce reliance on opioid analgesics during hospitalization due, at least in part, to the sedating effects of these pharmaceuticals. The rationale for this approach is that excessive sedation of rehabilitation 
JRRD, Volume 44, Number 2, 2007

Table 5.

Pain-related impairments and pain-management methods.

\begin{tabular}{lc}
\multicolumn{1}{c}{ Pain Variable } & $\mathbf{\%}$ \\
\hline Impairment & 42 \\
Recreational/Physical Activity & 34 \\
Emotional Functioning & 18 \\
Social Activity & 18 \\
Family Relationships & 14 \\
Sleep & 2 \\
Sexual Functioning & \\
Management Method & 100 \\
Medication & 40 \\
Physical Therapy & 38 \\
Occupational Therapy & 13 \\
Cognitive-Behavioral Therapy & 8 \\
Nerve Blocks & 6 \\
Transdermal Electrical Nerve Stimulation & 4 \\
Medication Pump Implantation & \\
\hline \hline
\end{tabular}

participants can result in reduced participation in therapies and further clouding of cognitive functioning in patients with TBI. Despite this decrease in opioid use, the average pain intensity rating at discharge (3.7) was significantly lower than the average admission rating [5.6; $\left.F_{(1,27)}=6.681, p=0.02\right]$.

In addition to the data regarding pain and painrelated problems in these injured soldiers, some information is available regarding the mental health problems of and treatment provided to these patients. In this sample, 78 percent of the injured military personnel experienced mental health problems during their time at the Tampa PRC. The most common mental health problems were posttraumatic stress disorder (PTSD) (44\%), adjustment disorders (38\%), and depressive disorders (24\%). For these mental health problems, the OEF and OIF returnees were provided with multiple mental health treatments, including medication management (64\%) and group (59\%) and individual (49\%) psychotherapy. Table 6 presents more complete data regarding the mental health problems and associated treatments in this sample. This information represents composite data for these military personnel and includes those with multiple psychological diagnoses. Unfortunately, limitations of this data set did not allow us to distinguish between emotional problems that were pain-related and those that were concurrent but not necessarily pain-related.

These data have several implications for PRC pain care. First, pain problems are almost universal among these war-injured patients, which highlights the need for
Table 6.

Mental health diagnoses and treatment.

\begin{tabular}{lc}
\hline Mental Health Variable & \% \\
\hline Diagnosis & 44 \\
Posttraumatic Stress Disorder & 38 \\
Adjustment Disorder & 24 \\
Depressive Disorder & 4 \\
Acute Stress Disorder & 4 \\
Substance Abuse & \\
Treatment Method & 64 \\
Medication Management & 59 \\
Group Psychotherapy & 49 \\
Individual Psychotherapy & 13 \\
Family Therapy & \\
\hline \hline
\end{tabular}

PRCs to consistently assess and treat pain. Second, although pain may resolve for some during their PRC stay, for most it does not, which indicates the need for ongoing treatment across the continuum of rehabilitation care. Third, contrary to expectations, pain-intervention procedures (e.g., epidural steroid injections, PCAs, stimulator implants) are less in demand in the rehabilitation setting than anticipated. In part, this finding may reflect the high proportion of TBI and associated cognitive impairment in this cohort, with associated concerns regarding the potential for negative patient reactions to invasive procedures. Fourth, the frequency of painrelated impairment in social and emotional functioning was surprisingly high given the relatively brief duration of pain that typifies these soldiers, and when combined with the high rates of mental health problems, suggests potential risks for the development of chronic pain syndromes as well as the need for multidisciplinary approaches to treatment that incorporate both medical and behavioral pain specialists.

\section{CHALLENGES TO PAIN CARE}

While polytraumatic injuries are not unique to combat casualties, and their successful management has long been a focus of trauma medicine [23], the rapid influx of severely wounded OEF and OIF theater personnel has heightened our awareness of several challenges to current perspectives in the field of pain management. Traditional pain-assessment practices may be less informative when applied to this population. Additionally, existing conceptualizations of the continuum of pain and its association with tissue damage may not apply fully to the course of healing 
experienced by the combat wounded and may require modification. Similarly, contemporary pain-care models may not meet the needs of this group of war-injured because of the extent of their physical and, at times, emotional wounds. We will discuss the primary challenges we have observed and, where possible, suggest potential modifications to these views which we believe may lead to improved pain services to the combat wounded.

\section{Pain Assessment}

Comprehensive assessment and reassessment of pain is the cornerstone of optimal pain management for all pain conditions. Because of the severe and multiple injuries usually present in the combat-wounded soldier, pain assessment is especially challenging. The battlefield environment is highly stressful, rapidly evolving, and emotionally charged because of the intensity of the conflicts in Iraq and Afghanistan. The frontline assessment of combatwounded soldiers focuses on triage, resuscitation, damage control, and stabilization, with the goal of rapid evacuation to a higher level of care. Often, a detailed pain assessment and pain-care plan are not addressed until the soldier is stabilized and evacuated. However, even following evacuation, the care of traumatic injuries or other serious conditions takes precedence and pain-related issues may be neglected or minimized.

Assessments may be further complicated when patients experience pain in multiple body regions. Edwards proposed a taxonomy to describe three categories of posttraumatic injuries: (1) regionalized (confined to one or a limited number of body regions, such as leg and knee), (2) broadly regionalized (peripheral injuries are bilateral or injuries involve the trunk), and (3) generalized (injuries involving large body regions) [10]. The preponderance of broadly regionalized or generalized injuries sustained by OEF and OIF combat wounded requires more detailed and lengthy site-specific pain assessments. Additional assessment complications arise from the various injury mechanisms involved (i.e., blast, penetrating, blunt, or burn). These insults may activate multiple pain pathways (nociceptive and neuropathic) and result in occult injuries to internal organs (e.g., rupture of a hollow viscous) where pain is poorly localized. Furthermore, those with combat casualties may be reticent to report pain because they view pain as a weakness or fear they might be perceived as weak by their fellow soldiers if they complain [24]. Finally, soldiers with TBI, especially those with cognitive impairment, pose additional difficulties for pain assess- ment. These soldiers may be unable to effectively and reliably communicate their pain because of confusion, limited consciousness, or injury-related deficits in sensory and motor functions. In the individual with decreased consciousness, changes in blood pressure, heart rate, pupil size, diaphoresis, and respiratory rate may be useful for estimating pain levels and guiding treatment, but vital signs are imperfect correlates of pain and may be affected by a variety of non-pain-related factors. Among those whose consciousness is normal, cognitive impairment-as reflected by slowed information processing, decreased memory, and poor attention-may impede pain assessment. Additionally, evidence from studies with elderly populations shows that nursing staff assess and document pain less frequently when patients are cognitively impaired [25-26] and that nursing staff assess pain less frequently and rate it as less severe as cognitive limitations increase [26]. Furthermore, when pain assessments are completed with nonvalidated instruments, associations between verbal pain scores of patients with cognitive impairment and instrument-based scores may be unrelated or even inversely related [27].

As the majority of polytrauma evacuees have symptoms of TBI (http://www.dvbic.org/), special strategies for pain assessment are needed that include an objective assessment of consciousness, cognition, and behavior [28]. A multitude of scales are available for assessing pain in the cognitively challenged, but few provide supporting psychometric data. For example, Stolee and colleagues reviewed 39 recent instruments and reported that none met contemporary validity and reliability standards [29]. Behavioral observations alone are not sufficient despite associations between pain and aggression [30] or agitation [31] in those with cognitive impairment because behavioral observations cannot discriminate between the multiple possible etiologies. Because studies comparing the reliability of self-reported pain scores across levels of cognitive impairment have found that approximately one-third of older adults with severe cognitive impairment could provide useful and valid pain ratings [32-33], providers should first attempt to elicit self-reports of pain using simple pain scales (e.g., the Faces Scale) for all responsive polytrauma patients. If reliable self-reports of pain are not possible, practitioners should rely on a combination of behavioral observations, knowledge of the pathophysiology of pain, trauma experience, analgesic effects, and family or significant other input when assessing pain in these individuals as outlined in the Veterans Health Administration National Pain Management Strategy 
Coordinating Committee's Consensus Statement "Assessing Pain in the Patient with Impaired Communication" (http://www.vachronicpain.org/). Because of the interaction between pain and function [34-35], evaluations should include, in addition to pain severity measures, measures or estimates of pain-related impairment in activity, emotional, social, familiar, sexual, and vocational domains of functioning whenever possible.

\section{Pain Classification}

The complex nature of combat-trauma pain poses a challenge to the clinical utility of the current pain classification system. In the current system, acute pain is viewed as an adaptive response that alerts the individual to the presence of a noxious stimulus or ongoing tissue damage. With normal tissue injury, the intensity of acute pain is a function of body tissue damage and the resultant activation of nociceptors at the site of the injury. If the body cannot restore physiological functions to normal homeostatic levels (i.e., cannot heal) because of the extent of the trauma or the occurrence of repeated insults, chronic pain will develop.

The most widely accepted definition of chronic pain is pain without apparent biological value that has persisted beyond normal tissue-healing time, which is usually considered to be 3 months [36]. Although this definition allows for the longer tissue-healing times required by combat-related traumatic injuries, the expected courses of healing for each injury site are not likely to coincide in such a manner that the patient's pain could be classified as simply acute or chronic. Given the high probability of multiple concurrent pain conditions following combat trauma, each potentially having a unique etiology; onset; and course, the current classification of pain into acute and chronic types may prove too narrow for facilitating comprehensive conceptualization and intervention.

Current biomedical theories describing the development of chronic pain focus on the neuronal plasticity of the peripheral and central pain pathways [37-38]. Both inflammatory nociceptive processes and neuropathic pain from injury to the nervous system have been found to contribute to the hyperexcitability and central sensitization that are hypothesized to cause the transition to a chronic pain state. In the case of polytrauma, these processes probably occur at least somewhat independently for different injury sites, potentially starting as early as the acute phase of care. In addition, for those individuals who have sustained injuries that require repeated surgical revision, the potential for additional nerve damage and prolonged inflammatory pain is significantly heightened. Further complications stem from the fact that some of the neurobiological changes that characterize chronic pain occur within hours of an acute injury [39]. Consequently, both acute and chronic pain processes may exist simultaneously in the combat-trauma patient. Because intervention objectives and strategies for these two types of pain may differ, adopting a more flexible approach to classification and conceptualization that allows concurrent and complementary treatment of the patient's pain conditions may be advantageous.

As a first step toward this end, we propose that the current classification approach be modified to include a transitional stage, postacute, between the acute and chronic stages in polytraumatic injuries (Figure). Although previous models have proposed various intervening stages between acute and chronic pain (e.g., recurrent acute pain), the recognition of a postacute stage in the context of polytrauma calls attention to the indeterminate nature of the complex constellation of pain states that characterizes the prolonged period of healing and ongoing medical interventions typically required. Focusing attention on this transitional stage may facilitate the targeted application of a range of pain interventions that have different objectives and expected outcomes. During the postacute stage, which is likely to characterize the rehabilitation phase of care, more aggressive pain-intervention strategies may prevent more complete transition to a chronic state. Additionally, the presence and treatment of acute pain during this transitional stage would not preclude the use of strategies traditionally reserved for chronic conditions should the individual exhibit indications of a transition to chronic pain. Intervention for recent-onset chronic pain, such as multidisciplinary rehabilitation, could then be implemented so the

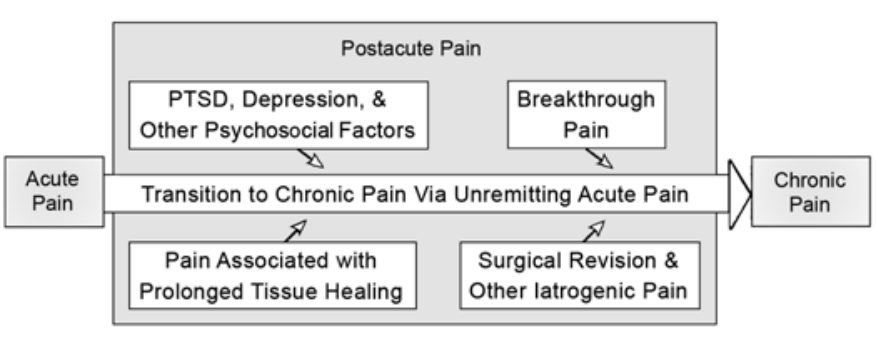

Figure.

Proposed revision in pain classification for combat-trauma pain. PTSD = posttraumatic stress disorder. 
development of pain-related disability and psychosocial distress could be prevented.

Because of the unique nature of combat-trauma pain, we do not know which patient and environmental characteristics will be important factors in the evolution of the pain condition. A number of risk factors have been identified in the development of chronic pain and associated disability among individuals with more limited injuries and medical conditions, but it remains to be seen if these associations will be the same among wounded soldiers. Although the most potent predictors of many chronic pain syndromes appear to be psychosocial variables [40], biomedical variables may be equally important determinants of risk in the polytrauma population. Available evidence suggests that medical factors such as site and extent of surgery (thoracic, major limb amputation, and spinal); the occurrence of postoperative infection, bleeding, organ rupture, compartment syndrome or unrelieved pain; and the need for multiple operations are all predictors of the development of chronic pain [41-42]. Biomedical factors such as these may play a relatively stronger role in the transition to chronic pain, while psychosocial variables such as posttraumatic stress reactions [43-44], depression [40], and pain-related fear of activity [45-47] may retain their primacy among the risk factors for pain-related disability.

We do not yet know what percentage of combatinjured patients who develop chronic pain will exhibit the symptoms of psychosocial distress that characterize many of the more severe pain syndromes. Although their youth, the potential for return to active duty, and the availability of rehabilitation services may serve as protective factors, many are likely to experience significant disability associated with their ongoing pain conditions. In addition to the psychosocial variables traditionally implicated in this process, many of these individuals will have the added challenges of adapting to significant loss of physical/ cognitive function. Until empirical data regarding these issues become available, practitioners should screen each trauma patient for the presence of pain-related psychosocial distress and refer those with evidence of these symptoms to qualified pain or behavioral-health specialists. Additionally, care providers should evaluate each patient for symptoms of mixed-pain conditions and if present, consider more aggressive and comprehensive strategies to manage each pain component.

\section{Pain Treatment}

The influx of OEF and OIF combat-injured patients into the military and VA healthcare systems has taxed existing resources and knowledge. Currently, a variety of barriers to providing state-of-the-art emergency and acute pain care to theater casualties are present. Most notably, pain data for wounded soldiers receiving emergent care is lacking, and barriers to data sharing between the military and the VA impede efforts to evaluate the longterm effectiveness of MTF pain-management technologies. Efforts to raise military medical staff awareness of the importance of assessing and aggressively treating pain are underway, and the use of advanced pain-management technologies and innovative methods is increasing. However, considerable room for improvement remains.

Future directions for improved pain management during the emergency and acute phases may involve locating acute pain service teams further forward on the battlefield at combat-support hospitals in key air-evacuation nodes. Other medications [48], medication combinations [49], pain-medication delivery methods [50], and paincontrol technologies [51] also are being explored. Using an Internet-based clinical tracking system called the Regional Anesthesia Tracking System, the MARAA committee will have the first extensive database of information on wounded-soldier pain and pain management during the war. Full utilization of this important information, which will have a significant effect on all trauma patients, will require increased cooperation and coordination between military medicine, VA, and civilian care providers. The knowledge gained in the current conflicts in managing trauma pain in wounded soldiers will likely translate into improved pain management for civilian trauma patients during times of national disaster or terrorist attack. This effort to manage trauma pain as a disease process during the emergency and acute phases of traumatic injury, rather then just a consequence of trauma, is one of the defining medical achievements of the 21st century.

Pain care during the rehabilitation phase faces a similar variety of challenges that reflect patient, care provider, therapy, and system factors. Patient factors include the high rates of head injury, cognitive impairment, and mixed-pain conditions that complicate pain assessment and treatment; the frequent necessity for repeated surgeries and subsequent episodic pain during rehabilitation; the presence of emotional conditions (e.g., PTSD) that may be triggered by pain or exacerbate pain; the pain-related 
fear that can lead to avoidance of the increased activity necessary for rehabilitation; and family beliefs about pain or pain treatment that can impede pain-management efforts. Provider factors include inconsistencies in pain assessment and pain documentation, lack of knowledge necessary to guide pain assessment in the cognitively impaired, prescription of insufficient analgesics that results in poorly controlled pain, and prescription of excessive analgesics that leads to sedation and interference with rehabilitation. Therapy issues include the need to address multiorgan medical problems while simultaneously treating pain and the frequent need for extended rehabilitation stays and/or multiple rehabilitation admissions, which require persistent and intensive pain-management efforts. System factors involve the need to make adequate pain-treatment resources available; to educate PRC staff regarding pain assessment, documentation, and treatment alternatives; and to develop integrated and consistent methods to assess PRC outcomes throughout the continuum of care.

To successfully address these issues will require continued innovative, comprehensive, and interdisciplinary efforts. One such effort has been the recent establishment of a National Polytrauma Pain Subcommittee with representation from all four inpatient PRCs. The goal of this group is to enhance PRC pain care by improving consistency, providing staff education, developing documentation and clinical tools, and promoting uniform outcome assessments. Fortunately, PRC pain care is evolving rapidly and will likely continue to progress and expand in concert with the development of the 23 Polytrauma Network Sites that the VA has authorized. PRC priorities for the future may include educating care providers in pain assessment, documentation, and treatment approaches; supporting efforts to increase the availability of pain specialist services; and developing pain clinical resources to ease provider burden and promote improved pain care.

\section{IMPLICATIONS FOR RESEARCH}

The severity and multiplicity of wounds suffered by the OEF and OIF combat injured highlight the scarcity of and limitations in the empirical data regarding traumarelated pain care. Numerous areas of needed research exist that are essential for developing, evaluating, and implementing effective and efficient pain-treatment approaches. Perhaps most important among all research issues is the need for alternative methods for accurately assessing pain in the soldier with cognitive impairment. Because all decisions regarding pain treatment during all phases of care evolve from this starting point and the majority of evacuated casualties present with TBI and associated cognitive limitations, alternative but reliable pain-assessment techniques are paramount for implementing and assessing the efficacy of pain interventions. Such assessments may be an even greater priority for PRCs, where active rehabilitation participation on the part of the patient is required and optimal pain control must provide relief but not excessive sedation. As indicated previously, although several observational instruments have been promoted for use with this population, they lack adequate validation data.

A second research priority that affects pain care across all trauma phases is the need to develop a systematic and standardized method of assessing pain outcomes. In contrast to the past, when trauma care research focused almost exclusively on survival, future research should include emotional, cognitive, functional, and social outcomes variables that better reflect the broad and protracted effects of trauma [52]. Availability of this information would allow informed decisions about treatments that maximize pain outcomes, while concomitantly addressing potential gaps in contemporary pain-classification systems. Perhaps most importantly, these data would facilitate efforts to empirically evaluate risks of chronic pain development and chronic pain syndromes among these severely wounded soldiers, project future resource needs to deal with these problems, and identify what, if any, treatment approaches can reduce risks for transition into costly and disabling chronic pain conditions. Similar calls for research have been voiced regarding risk factors for the development of chronic postsurgical pain [53].

Other research priorities should target the development and evaluation of education or policy initiatives that improve the consistency of pain assessment and treatment practices across the continuum of care. During all phases of care, medical staff need to become more adept at assessing and documenting pain in the severely wounded as a precursor to providing effective pain care. Similarly, educational endeavors targeting improved communication between categories of providers (i.e., field and MTF military medical staff, VA PRC staff, and community practitioners) that enhance continuity and effectiveness of pain care are needed. Finally, given the unique combination of pain, traumatic stress, and psychosocial factors that characterize these individuals, methods that encourage the adoption of interdisciplinary 
approaches designed to provide "total pain care" need to be implemented, particularly during the rehabilitation phase.

\section{CONCLUSIONS}

The healthcare demands associated with the increasing numbers of severely injured OEF and OIF soldiers are growing. At present, acute care needs can be loosely predicted based on prior OEF and OIF casualty data. What remains unknown is the extent to which long-term disability will develop among recovering military personnel. While Persian Gulf war data may provide some estimates of the likely prevalence of chronic physical and emotional conditions, the protracted nature of the OEF and OIF conflicts, the increased risk exposure because of constant threats, the lengthy deployment periods and multiple deployment episodes, the preponderance of blast injuries with their unknown long-term consequences, and the enhanced survival rates among even the most grievously wounded pose new challenges perhaps unmatched by any previous conflict, including Vietnam. Current data from soldiers with nonsevere injuries registering for VA care indicate that pain problems will be among the most common complaints for all returning military and that pain prevalence may surpass rates observed following the Persian Gulf war [54-55]. Among combat-trauma cases, pain will continue to be an almost universal occurrence with consequences for both current and future morbidities.

Fortunately, both the military and the VA are responding swiftly to the pain-care needs of wounded soldiers. Innovative pain-care strategies have been instituted during all phases of casualty care, and continued evolution of these efforts is certain. Indeed, the VA's responsibility to provide rehabilitation services to the most severely wounded soldiers is perhaps fortuitous given the department's wealth of experience and expertise in the delivery of intensive, interdisciplinary care. Unfortunately, in many cases, clinical care has outpaced associated research, which results in considerable uncertainty about the long-term efficacy of these necessary, but untested, pain-care efforts. We hope that this gap between practice and research will narrow rapidly and provide trauma and rehabilitation staff with effective and efficient methods to combat pain and its associated sequelae.

\section{ACKNOWLEDGMENTS}

All opinions expressed herein are those of the authors and do not in any way represent those of the Department of Defense or the VA.

This material was based on work supported by the VA Rehabilitation Research and Development Service (grant O3283R to M. Clark and grant B4005R to R. Gironda).

The authors have declared that no competing interests exist.

\section{REFERENCES}

1. Veterans Health Administration. VHA Handbook 1172.1. Part 2(a). Washington (DC): Department of Veterans Affairs; 2005. p. 1.

2. Gawande A. Casualties of war-Military care for the wounded from Iraq and Afghanistan. N Engl J Med. 2004; 351(24):2471-75. [PMID: 15590948$]$

3. Gondusky JS, Reiter MP. Protecting military convoys in Iraq: An examination of battle injuries sustained by a mechanized battalion during Operation Iraqi Freedom II. Mil Med. 2005;170(6):546-49. [PMID: 16001610]

4. Patel TH, Wenner KA, Price SA, Weber MA, Leveridge A, McAtee SJ. A U.S. Army Forward Surgical Team's experience in Operation Iraqi Freedom. J Trauma. 2004;57(2): 201-7. [PMID: 15345962]

5. Peoples GE, Gerlinger T, Craig R, Burlingame B. The 274th Forward Surgical Team experience during Operation Enduring Freedom. Mil Med. 2005;170(6):451-59. [PMID: 16001590]

6. Grissom TE, Farmer JC. The provision of sophisticated critical care beyond the hospital: Lessons from physiology and military experiences that apply to civil disaster medical response. Crit Care Med. 2005;33(1 Suppl):S13-21. [PMID: 15640673]

7. Braganza RJ. Pain of trauma. In: Warfield CA, Fausett HJ, editors. Manual of pain management. 2nd ed. Philadelphia (PA): Lippincott Williams \& Wilkins; 2002. p. 231-34.

8. Osgood PF, Szyfelbein SK. Management of pain. In: Martyn JA, editor. Acute management of the burned patient. Philadelphia (PA): Saunders; 1990. p. 201-16.

9. Gregory J, Jackson S. The impact of introducing patient controlled analgesia for trauma patients admitted through accident and emergency. J Orthop Nurs. 2004;8(2):91-95.

10. Edwards WT. Posttrauma pain. In: Loeser JD, Bonica JJ, editors. Bonica's management of pain. 3rd ed. Philadelphia (PA): Lippincott Williams \& Wilkins; 2001. p. 788-93. 
11. Belanger HG, Scott SG, Scholten J, Curtiss G, Vanderploeg RD. Utility of mechanism-of-injury-based assessment and treatment: Blast Injury Program case illustration. J Rehabil Res Dev. 2005;42(4):403-12. [PMID: 16320137$]$

12. Conti G, Costa R, Pellegrini A, Craba A, Cavaliere F. Analgesia in PACU: Intravenous opioids. Curr Drug Targets. 2005;6(7):767-71. [PMID: 16305448]

13. Buckenmaier CC 3rd, Lee EH, Shields CH, Sampson JB, Chiles JH. Regional anesthesia in austere environments. Reg Anesth Pain Med. 2003;28(4):321-27. [PMID: 12945026$]$

14. Thompson GE. Anesthesia for battle casualties in Vietnam. JAMA. 1967;201:215-19.

15. Buckenmaier CC, McKnight GM, Winkley JV, Bleckner LL, Shannon C, Klein SM, Lyons RC, Chiles JH. Continuous peripheral nerve block for battlefield anesthesia and evacuation. Reg Anesth Pain Med. 2005;30(2):202-5. [PMID: 15765463]

16. Grass JA. Patient-controlled analgesia. Anesth Analg. 2005;101(5 Suppl):S44-61. [PMID: 16334492]

17. Savoia G, Gravino E, Loreto M, Erman A. Analgesia in PACU: Indications, monitoring, complications. Curr Drug Targets. 2005;6(7):755-65. [PMID: 16305454]

18. Hinsley DE, Rosell PA, Rowlands TK, Clasper JC. Penetrating missile injuries during asymmetric warfare in the 2003 Gulf conflict. Br J Surg. 2005;92(5):637-42.

[PMID: 15744703]

19. Stojadinovic A, Auton A, Peoples GE, McKnight GM, Shields C, Cross SM, Bleckner LL, Winkley J, MoniscaloTheberge ME, Buckenmaier CC 3rd. Responding to challenges in modern combat casualty care: Innovative use of advanced regional anesthesia. Pain Med. 2006;7(4):330-38. [PMID: 16898944$]$

20. Grant SA, Nielsen KC, Greengrass RA, Steele SM, Klein SM. Continuous peripheral nerve block for ambulatory surgery. Reg Anesth Pain Med. 2001;26(3):209-14. [PMID: 11359219]

21. Klein SM, Nielsen KC, Martin A, White W, Warner DS, Steele SM, Speer KP, Greengrass RA. Interscalene brachial plexus block with continuous intraarticular infusion of ropivacaine. Anesth Analg. 2001;93(3):601-5. [PMID: 11524326]

22. Veterans Health Administration. VHA Handbook 1172.1. Part 3(i). Washington (DC): Department of Veterans Affairs; 2005. p. 3.

23. Almogy G, Belzberg H, Mintz Y, Pikarsky AK, Zamir G, Rivkind AI. Suicide bombing attacks: Update and modifications to the protocol. Ann Surg. 2004;239(3):295-303. [PMID: 15075644$]$

24. Machin P, De C Williams AC. Stiff upper lip: Coping strategies of World War II veterans with phantom limb pain. Clin J Pain. 1998;14(4):290-94. [PMID: 9874006]
25. Davies E, Male M, Reimer V, Turner M. Pain assessment and cognitive impairment: Part 2. Nurs Stand. 2004;19(13): 33-40. [PMID: 15624384]

26. Wu N, Miller SC, Lapane K, Roy J, Mor V. Impact of cognitive function on assessments of nursing home residents' pain. Med Care. 2005;43(9):934-39. [PMID: 16116359]

27. Tsai PF, Richards K. Using an osteoarthritis-specific pain measure in elders with cognitive impairment: A pilot study. J Nurs Manag. 2006;14(2):90-95. [PMID: 16487420]

28. Branca B, Lake AE. Psychological and neuropsychological integration in multidisciplinary pain management after TBI. J Head Trauma Rehabil. 2004;19(1):40-57. [PMID: 14732830]

29. Stolee P, Hillier LM, Esbaugh J, Bol N, McKellar L, Gauthier N. Instruments for the assessment of pain in older persons with cognitive impairment. J Am Geriatr Soc. 2005; 53(2):319-26. [PMID: 15673359]

30. Feldt KS, Warne MA, Ryden MB. Examining pain in aggressive cognitively impaired older adults. J Gerontol Nurs. 1998;24(11):14-22. [PMID: 10392090]

31. Zieber CG, Hagen B, Armstrong-Esther C, Aho M. Pain and agitation in long-term care residents with dementia: Use of the Pittsburgh Agitation Scale. Int J Palliat Nurs. 2005;11(2):71-78. [PMID: 15798498]

32. Closs SJ, Barr B, Briggs M, Cash K, Seers K. A comparison of five pain assessment scales for nursing home residents with varying degrees of cognitive impairment. J Pain Symptom Manage. 2004;27(3):196-205. [PMID: 15010098]

33. Manz BD, Mosier R, Nusser-Gerlach MA, Bergstrom N, Agrawal S. Pain assessment in the cognitively impaired and unimpaired elderly. Pain Manag Nurs. 2000;1(4):106-15. [PMID: 11709864]

34. Clark ME, Gironda RJ, Carter S. Assuring the quality of pain services: Assessing outcomes. In: Boswell MV, Cole BE, editors. Weiner's pain management: A practical guide for clinicians. 7th ed. Boca Raton (FL): CRC Press; 2006. p. 99-116.

35. Rehabilitation Accreditation Commission. Standards manual: Medical rehabilitation. Revised ed. Tucson (AZ): Rehabilitation Accreditation Commission; 2002.

36. International Association for the Study of Pain, Subcommittee on Taxonomy. Classification of chronic pain. Descriptions of chronic pain syndromes and definitions of pain terms. Pain Suppl. 1986;3:S1-226. [PMID: 3461421]

37. Baron R. Mechanisms of disease: Neuropathic pain-A clinical perspective. Nat Clin Pract Neurol. 2006;2(2):95106. [PMID: 16932531]

38. Vadivelu N, Sinatra R. Recent advances in elucidating pain mechanisms. Curr Opin Anaesthesiol. 2005;18(5):540-47. [PMID: 16534290] 
39. Perkins FM, Kehlet H. Chronic pain as an outcome of surgery. A review of predictive factors. Anesthesiology. 2000;93(4):1123-33. [PMID: 11020770]

40. Dersh J, Polatin PB, Gatchel RJ. Chronic pain and psychopathology: Research findings and theoretical considerations. Psychosom Med. 2002;64(5):773-86. [PMID: 12271108]

41. Shipton EA, Tait B. Flagging the pain: Preventing the burden of chronic pain by identifying and treating risk factors in acute pain. Eur J Anaesthesiol. 2005;22(6):405-12. [PMID: 15991501$]$

42. Sondenaa K, Nesvik I, Breivik K, Korner H. Long-term follow-up of 1059 consecutive primary and recurrent inguinal hernias in a teaching hospital. Eur J Surg. 2001; 167(2):125-29. [PMID: 11266252]

43. Asmundson GJ, Coons MJ, Taylor S, Katz J. PTSD and the experience of pain: Research and clinical implications of shared vulnerability and mutual maintenance models. Can J Psychiatry. 2002;47(10):930-37. [PMID: 12553128]

44. Bryant RA, Marosszeky JE, Crooks J, Baguly IJ, Gurka JA. Posttraumatic stress disorder and psychosocial functioning after severe traumatic brain injury. J Nerv Ment Dis. 2001;189(2):109-13. [PMID: 11225683]

45. Fritz JM, George SZ. Identifying psychosocial variables in patients with acute work-related low back pain: The importance of fear-avoidance beliefs. Phys Ther. 2002;82(10): 973-83. [PMID: 12350212]

46. Crombez G, Vlaeyen JW, Heuts PH, Lysens R. Painrelated fear is more disabling than pain itself: Evidence on the role of pain-related fear in chronic back pain disability. Pain. 1999;80(1-2):329-39. [PMID: 10204746]

47. Vlaeyen JW, De Jong J, Geilen M, Heuts PH, Van Breukelen G. The treatment of fear of movement/(re)injury in chronic low back pain: Further evidence on the effective- ness of exposure in vivo. Clin J Pain. 2002;18(4):251-61. [PMID: 12131067]

48. Doan BK, Caldwell JA, Hursh SR, Whitmore JN, O’Donnell $\mathrm{RD}$, Russo MB. Assessing psychoactive pharmaceuticals and transitioning pharmacological fatigue countermeasures into operational environments. Aviat Space Environ Med. 2005;76(7 Suppl):C34-38. [PMID: 16018328]

49. Trouwborst A, Weber BK, Dufour D. Medical statistics of battlefield casualties. Injury. 1987;18(2):96-99.

[PMID: 3508167]

50. Kotwal RS, O’Connor KC, Johnson TR, Mosely DS, Meyer DE, Holcomb JB. A novel pain management strategy for combat casualty care. Ann Emerg Med. 2004;44(2): 121-27. [PMID: 15278083]

51. Szycher M, Setterstrom JA, Vincent JW, Battistone G. Spandra: A sustained release battlefield wound dressing. J Biomater Appl. 1986;1(2):274-304. [PMID: 3333401]

52. Bouillon B, Neugebauer E. Outcome after polytrauma. Langenbecks Arch Surg. 1998;383(3-4):228-34. [PMID: 9776447]

53. Kehlet H, Jensen TS, Woolf CJ. Persistent postsurgical pain: Risk factors and prevention. Lancet. 2006;367(9522): 1618-25. [PMID: 16698416]

54. Clark ME. Post-deployment pain: A need for rapid detection and intervention. Pain Med. 2004;5(4):333-34. [PMID: 15563318]

55. Gironda RJ, Clark ME, Massengale JP, Walker RL. Pain among veterans of Operations Enduring Freedom and Iraqi Freedom. Pain Med. 2006;7(4):339-43. [PMID: 16898945]

Submitted for publication May 31, 2006. Accepted in revised form November 15, 2006. 